Discapacidad y de la Salud (CIF) 2001. Rev Esp Salud Pública 2002;76(4):271-9.

3. Muñoz V. El derecho a la educación de las personas con discapacidades. Informe del Relator Especial sobre el derecho a la educación. Aplicación de la Resolución 60/251 de la Asamblea General, del 15 de marzo de 2006. A/ $\mathrm{HRC} / 4 / 29 ; 2007$.

4. INDEC. La pobreza en la Argentina: Asociación de características de los hogares con mediciones de la pobreza y diseño de un indicador compuesto de Necesidades Básicas Insatisfechas. $2^{\text {da }}$ ed. Buenos Aires: INDEC; 1985;1(Suppl 1).

5. Mc Caffery M, Beebe A. Pain: Clinical Manual for Nursing Practice. Baltimore: U.V. Mosby Company; 1993.

6. Comité Nacional de Crecimiento y Desarrollo. Sociedad Argentina de Pediatría. Guías para la Evaluación del Crecimiento. $2^{\mathrm{da}}$ ed; Buenos Aires: SAP; 2001.

7. Consejo nacional de coordinación de políticas sociales. Programa de Accesibilidad para ámbitos educativos. [Consulta: 3 de abril de 2014]. Disponible en: http:/ / www. conadis.gov.ar/Programa\%20Ley\%20de $\% 20$ Cheques $\% 20$ $2014 \% 20$ Programa\%20de\%20Accesibilidad $\% 20$ para $\% 20$ ambitos\%20educativos.pdf.

8. Blanco R. La equidad y la inclusión social: uno de los de- safíos de la Educación y la Escuela hoy. Rev Electrón iberoam calid, efic cambio educ 2006;4(3):1-15.

9. Jiménez A, Huete A. La discriminación por motivos de discapacidad. Madrid: CERMI. 2002. [Consulta: 3 de abril de 2014]. Disponible en: http://www.cermi.es/ es-Es/ColeccionesCermi /Cermi.es/Lists /Coleccion/ Attachments/76/3disciminacion.pdf.

10. INDEC-UNICEF, Sistema Integradodeindicadores Sociales y Económicos sobre la niñez y la adolescencia en la Argentina (SIISENA); elaboración sobre la base de procesamientos especiales suministrados por la Primera Encuesta Nacional de Personas con Discapacidad 2002-2003, complementaria al Censo 2001.

11. Beech J, Larrondo M. La inclusión educativa en la Argentina de hoy. Definiciones, logros y desafíos a futuro. Argentina. Taller regional preparatorio sobre educación inclusiva. América Latina, Regiones Andina y ConoSur. Buenos Aires: UNESCO; 2007.

12. Muñoz V. Derecho a la educación de las personas con discapacidad en América Latina y el Caribe. Informe para la Comisión Interamericana de Derechos Humanos. Noviembre, 2009. [Consulta 3 de abril de 2014]. Disponible en: www. asdown.org/descargas/educacion_personas_con_discapacidad_informacion.pdf

\title{
Análisis del número y diseño de ensayos clínicos y de la prescripción de medicamentos para indicaciones no incluidas en su ficha técnica en pacientes hospitalizados pediátricos
}

\author{
Analysis of clinical trials and off-label drug use in hospitalized pediatric patients
}

\author{
Dra. Tamara Roldán $n^{a}$ Dra. Elena Villamañán ${ }^{a}$, Dra. Margarita Ruano ${ }^{a}$, Dra. Yolanda Larrubia ${ }^{a}$ \\ Dra. Pilar Gómez-Salcedo ${ }^{a}$ y Dra. Alicia Herrero ${ }^{a}$
}

\section{RESUMEN}

Introducción. La falta de ensayos clínicos en pediatría (ECP) conduce a la prescripción off-label de medicamentos en niños (POMN). Nuestro objetivo fue analizar el número y diseño de ECP y de POMN en los últimos años.

Población, material y métodos. Estudio observacional, retrospectivo de ECP y POMN desde 2007 hasta 2012 realizados en un hospital infantil con 252 camas. Se analizó el número y diseño de ECP y de POMN por año y sus características.

Resultados. Se evaluaron 87 ECP y 449 principios activos correspondientes a 1049 medicamentos prescritos a niños

a. Servicio de Farmacia. Hospital Universitario La Paz, Madrid, España.

Correspondencia:

Dra. Elena Villamañán: evillabueno@telefonica.net

Conflicto de intereses: Ninguno que declarar.

Recibido: 17-12-2013

Aceptado: 3-2-2014 hospitalizados. De ellos, $117(26 \%)$ se utilizaron fuera de prospecto. Los ECP fueron en aumento desde 2008 hasta 2011. Ese año, el 52,2\% de los ECP fueron no aleatorizados ni controlados y solo $39,1 \%$ fueron aleatorizados controlados. Un 77\% de los fármacos investigados eran prescritos fuera de prospecto. La POMN se mantuvo estable durante el estudio. Conclusiones. En nuestro hospital, ha aumentado la investigación en pediatría en los últimos años; los estudios no aleatorizados ni controlados fueron los más frecuentes. La POMN no se ha modificado.

Palabras clave: investigación, ensayos clínicos, prescripción de fármacos fuera de prospecto.

http:/ /dx.doi.org/10.5546/aap.2014.249

\section{INTRODUCCIÓN}

La prescripción de fármacos en niños, a menudo, se hace sin que hayan sido estudiados en ensayos clínicos para este grupo de pacientes. ${ }^{1}$ Según diversos estudios, hasta un $70 \%$ de los fármacos no tienen incluidos en su ficha técnica (FT) suficientes datos sobre la edad pediátrica. ${ }^{2}$ 
Entre un 36 y un $100 \%$ de los niños hospitalizados reciben tratamientos fuera de prospecto (off-label), ${ }^{3}$ los menores de 2 años y los neonatos son los que reciben mayoritariamente tratamientos en estas condiciones. $^{4}$

A pesar de ello, el desarrollo de ensayos clínicos en pediatría (ECP) es escaso. Existen barreras, como la dificultad de desarrollar estudios aleatorizados y controlados, problemas éticos o la menor proporción de pacientes. ${ }^{5}$ Además, en muchos casos, la industria farmacéutica no está interesada en invertir en ECP debido al alto costo y la escasa proporción de población potencial a la que van dirigidos. ${ }^{6}$

En este contexto, con el fin de incentivar la investigación de fármacos en pediatría, las distintas instituciones de la salud han puesto en marcha nuevas iniciativas. Así, la Organización Mundial de la Salud emitió la resolución WHA60.20,7 que urgía a sus 163 miembros al desarrollo de medidas para mejorar la disponibilidad de fármacos para niños. En 2003, en EE.UU., se promulgó una nueva ley (Pediatric Research Equity Act), actualmente vigente, ${ }^{8}$ y en Europa, en 2006, el Parlamento europeo publicó el Reglamento Pediátrico. ${ }^{9}$

El siguiente estudio fue realizado con el objetivo de evaluar el número y diseño de los ECP y de las prescripciones fuera de la ficha técnica de medicamentos en niños hospitalizados en los últimos años.

\section{MATERIAL Y MÉTODOS}

Estudio observacional, retrospectivo, llevado a cabo de 2007 a 2012. El trabajo se desarrolló en España, en un hospital público, terciario y universitario, localizado en Madrid, que comprende un hospital infantil con 252 camas, 28 subespecialidades y atiende a cerca de 9000 niños ingresados al año.

Se incluyeron todos los ECP llevados a cabo en el centro y todos los medicamentos prescritos a los niños hospitalizados durante el período evaluado. Se analizó el número y diseño de ECP realizados por año y el número de fármacos utilizados para tratar a niños hospitalizados, no en investigación y prescritos fuera de prospecto cada año.

En relación con las características de los $\mathrm{ECP}$, se recogieron los siguientes dominios, como figuran en el documento de aprobación de la European Medicines Agency (EMA): rango de edad, características del diseño (fase del estudio, si era abierto o ciego, aleatorizado o no y si era controlado o no), fármacos implicados y especialidades pediátricas.

Para valorar las condiciones de uso de los fármacos prescritos, de acuerdo con las indicaciones recogidas en su FT, establecimos tres categorías: 1) no evaluado (la eficacia y seguridad no han sido establecidas en la población infantil); 2) no autorizado (uso contraindicado en la población pediátrica); 3) no especificado (no

FIgURA 1. Relacción de ensayos clínicos en pediatría respecto a los ensayos clínicos totales iniciados en el Servicio de Farmacia

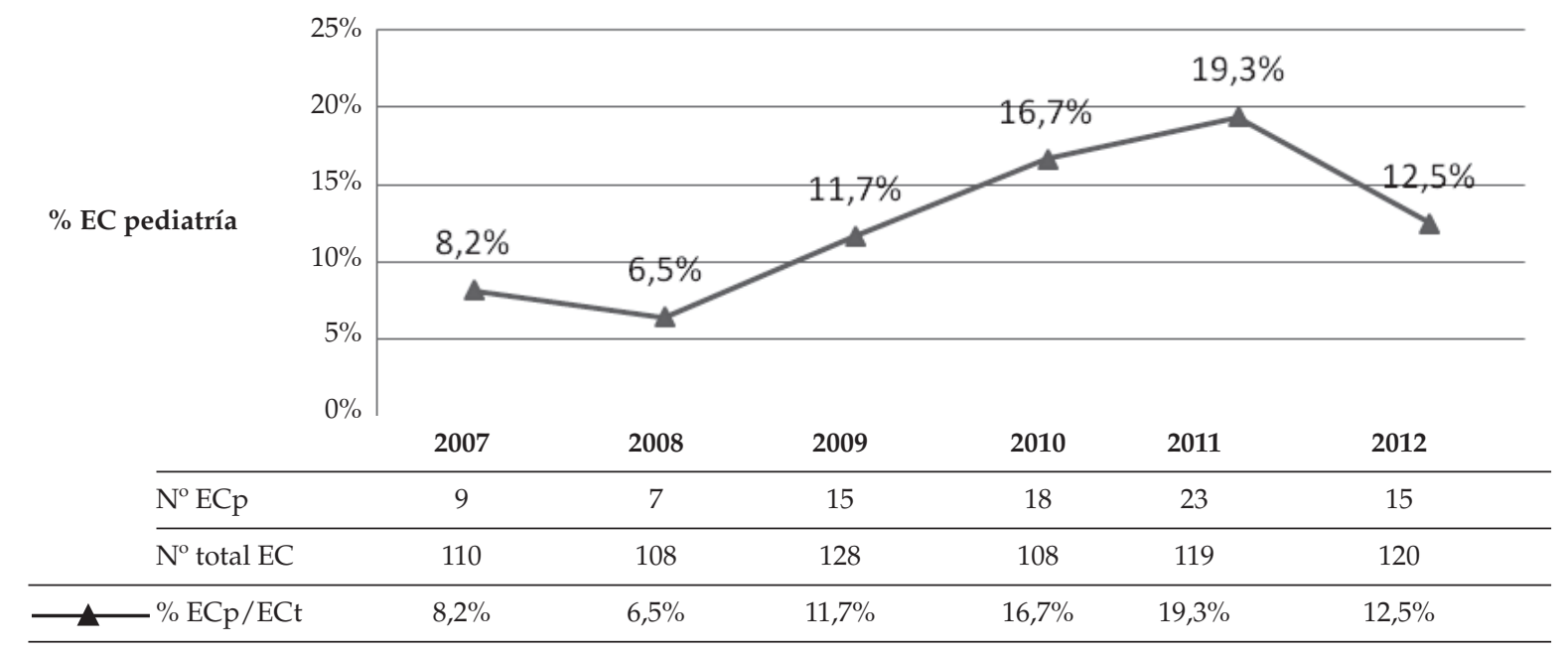

EC: ensayos clínicos; ECp: ensayos clínicos en pediatría; ECt: ensayos clínicos totales. 
existe información específica para la población pediátrica en su FT). También evaluamos el tipo de fármacos prescritos a niños fuera de prospecto y especialidades.

Las fuentes de datos para el estudio fueron, por un lado, el programa informático de gestión de ensayos clínicos del hospital, aplicación creada ad hoc por la unidad de informática del centro en la que se recopilan las características de los ensayos clínicos de acuerdo con el registro de aprobación de la EMA, y, por otro lado, el programa de prescripción electrónica (PE) FarmaTools ${ }^{\circledR}$ (versión 2.5), a través del cual se evaluaron las prescripciones de medicamentos a niños hospitalizados. En este programa, se registran, entre otros datos, la población a la que se prescribe, la dosis que se utiliza, el intervalo interdosis o la indicación.

El trabajo recibió la autorización del Comité de Ética en Investigación Clínica del centro.

\section{RESULTADOS}

Se analizaron 87 ECP y 449 principios activos, correspondientes a 1049 medicamentos comercialmente disponibles prescritos a niños hospitalizados. De ellos, 117 (26\%) fueron POMN.

El número de ECP se incrementó desde 2008 hasta 2011 y alcanzó casi un 20\% del total de los ensayos clínicos realizados en el centro, que incluyeron adultos y niños (Figura 1). Paralelamente, la POMN, a lo largo del período de estudio, se mantuvo estable y siempre por encima del 20\%, como muestra la Figura 2.

Los ECP analizados fueron, en su mayoría $(64,4 \%)$, estudios en fase III seguidos de los estudios en fase II ( $23 \%)$, principalmente abiertos
(71\%), no aleatorizados $(58,1 \%)$ y no controlados $(61,3 \%)$. Los estudios aleatorizados, ciegos y controlados prevalecieron hasta 2008 sobre los abiertos no aleatorizados y no controlados, y llegaron a alcanzar un 57,1\% del total de ECP. Entre 2008 y 2009, se produjo un cambio de tendencia y aumentaron los estudios abiertos no aleatorizados ni controlados, aunque, de manera global, la evolución de ambos tipos de diseño permaneció estable. Al final del período de estudio, el número de ambos tipos de ECP se aproximó bastante; supuso un 33,3\% del total de ECP y un $26,7 \%$, respectivamente (Figura 3 ).

En cuanto a los fármacos investigados en $\mathrm{ECP}$, un $77 \%$ de los casos fueron medicamentos prescritos fuera de prospecto y un $23 \%$, fármacos experimentales no comercializados. Se investigaron, sobre todo, antiinfecciosos $(34,5 \%)$, antineoplásicos e inmunomoduladores $(20,7 \%)$ y fármacos cardiovasculares (11,5\%). La evolución de los ECP con estos grupos terapéuticos a lo largo del estudio se muestra en la Figura 4.

Las especialidades pediátricas que llevaron a cabo más ECP fueron hematooncología (19,5\%) y neonatología (11,5\%). Todos los ECP incluidos en el estudio fueron multicéntricos y el promotor, en todos los casos, fue la industria farmacéutica.

Al analizar las condiciones de prescripción en pediatría, observamos que un $80,8 \%$ de los medicamentos correspondieron a la categoría $a$ (no evaluado/no recomendado; la eficacia y la seguridad no han sido establecidas en la población infantil); un $15,4 \%$, a la $b$ (no autorizado; indicación de uso contraindicada para la población pediátrica); y un 3,8\%, a la c (no especificado en la FT; no existe información sobre

FIgURA 2. Evolución del consumo de medicamentos según las condiciones de uso

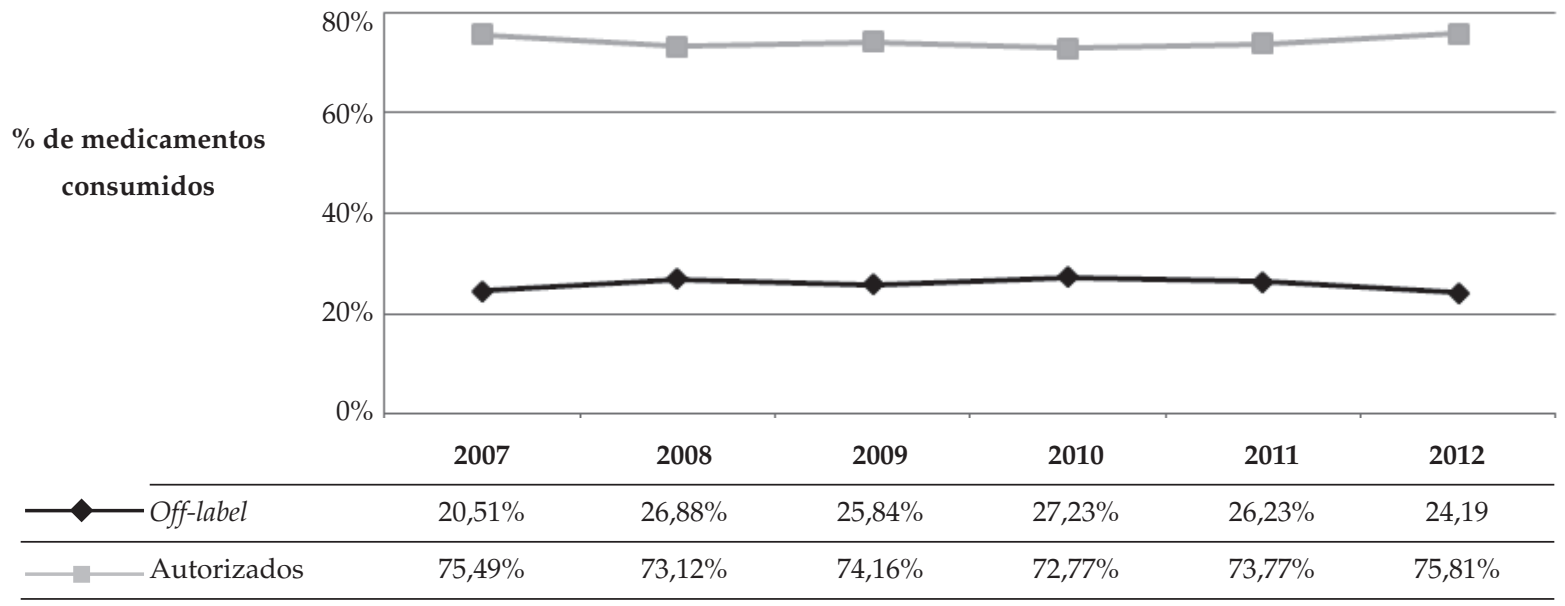


la población pediátrica).

El mayor número de medicamentos utilizados rutinariamente en pediatría correspondió a terapia del aparato digestivo $(25,6 \%)$, antiinfecciosos $(21,8 \%)$ y sistema nervioso $(16,5 \%)$. Sin embargo, los grupos terapéuticos utilizados con más frecuencia fuera de prospecto fueron hipnóticos y sedantes (lormetazepam y clometiazol) en un $5,73 \%$, antitrombóticos (enoxaparina, dipiridamol y bemiparina) en un $2,95 \%$ y antibacterianos (daptomicina) en un 1,27\%.

Por especialidades pediátricas, las que prescribieron más medicamentos fuera de la ficha técnica fueron pediatría general $(28,8 \%)$, cirugía pediátrica $(19,5 \%)$, unidad de trasplante hepatorenal $(15,5 \%)$, hematooncología $(11,0 \%)$ y neonatología $(4,7 \%)$.

\section{DISCUSIÓN}

Según nuestros resultados, y en concordancia con otros estudios realizados, ${ }^{10}$ desde 2007 hasta 2011, se produjo un incremento del número de ECP, hecho que podría estar relacionado con la publicación en Europa en 2006 de nuevas iniciativas para incentivar la investigación de fármacos en niños. ${ }^{9}$

Sin embargo, la prescripción de medicamentos fuera de prospecto en pediatría se mantuvo

FIgURA 3. Evolución de los ensayos clínicos según el diseño

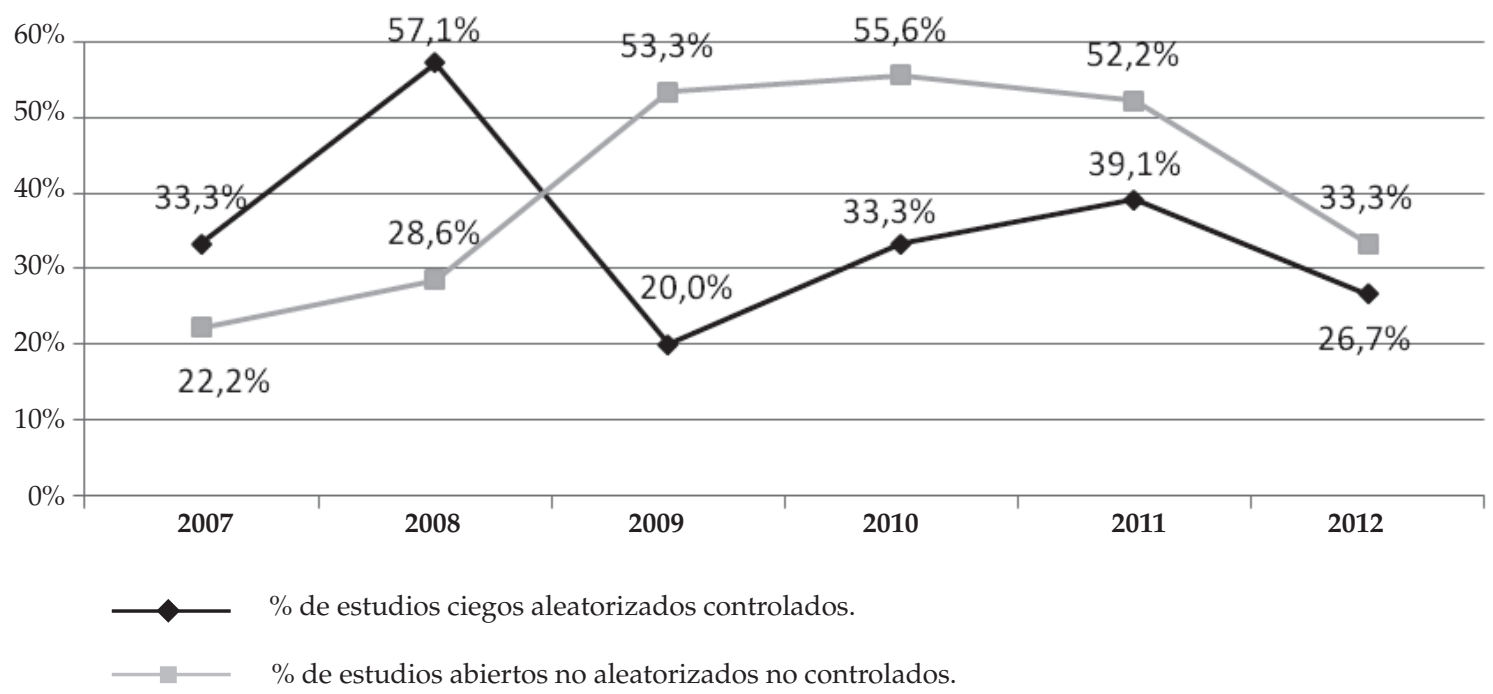

FIgURA 4. Evolución de los ensayos clínicos en pediatría por grupo terapéutico

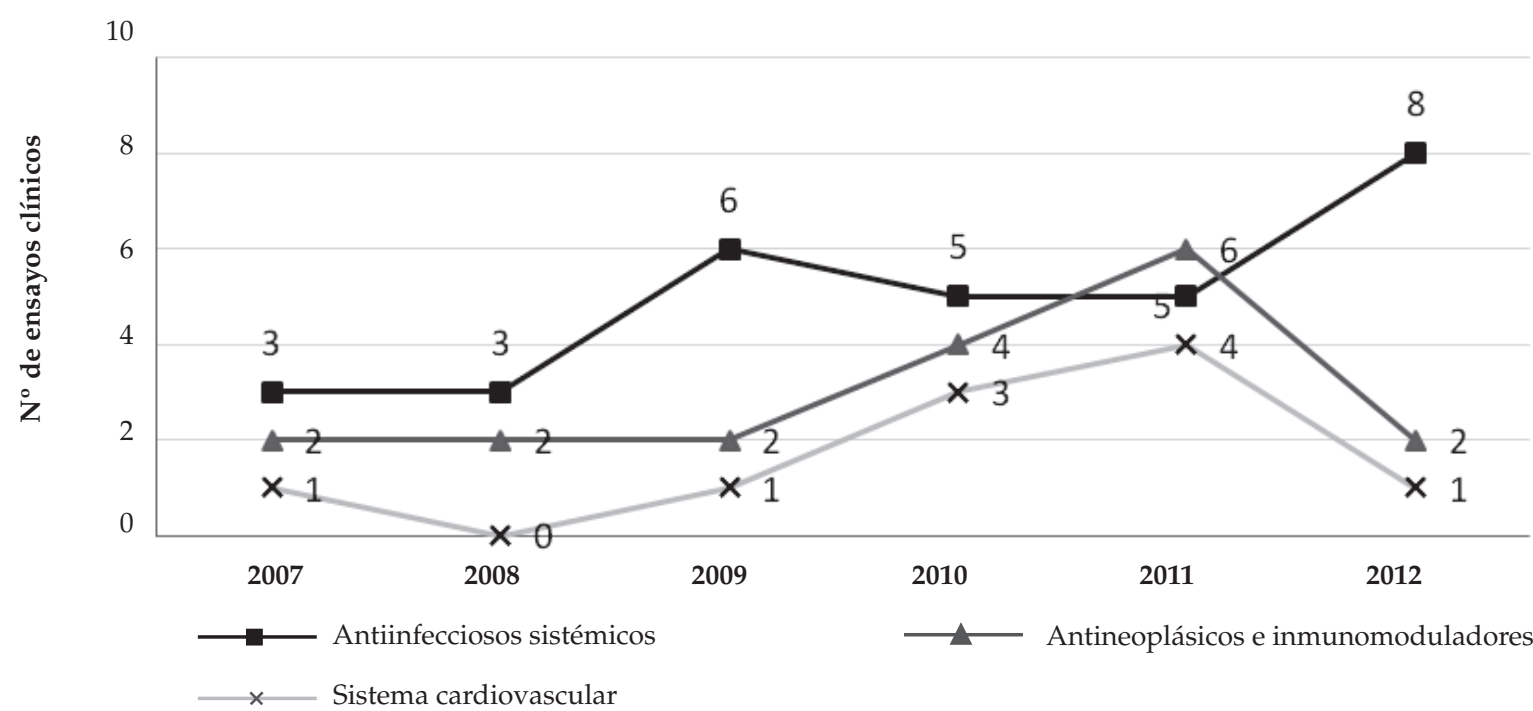


más o menos estable a lo largo del estudio. Los resultados obtenidos muestran que más de la cuarta parte de los medicamentos se utilizaron en estas condiciones y se debió fundamentalmente a la falta de estudios en pediatría, según se especifica en sus FT. Otros autores han obtenido resultados también en esta línea y han detectado tasas parecidas e incluso más altas. ${ }^{11,12}$

En cuanto al diseño, llama la atención que los ECP han cambiado notablemente durante los últimos años en nuestro centro: pasaron de ser en su mayoría aleatorizados y controlados a casi igualarse al número de estudios abiertos no controlados y no aleatorizados. Como es sabido, los ensayos clínicos aleatorizados y controlados constituyen la mejor herramienta para evaluar la eficacia y la seguridad de un tratamiento, pero los estudios abiertos no controlados suponen una menor complejidad e inversión económica. En este sentido, los incentivos a la investigación podrían haber influido ejerciendo un efecto positivo en términos cuantitativos, pero negativo en cuanto a su calidad.

En concordancia con otros trabajos,$^{13}$ la mayor parte de los fármacos implicados en ECP fueron antiinfecciosos, los cuales figuran, además, entre los fármacos considerados prioritarios para su investigación en niños según la EMA. ${ }^{14}$

Por especialidades, hematooncología y neonatología llevaron a cabo un mayor número de ECP, probablemente debido a la gravedad y a la falta de alternativas terapéuticas disponibles para estos pacientes.

Los medicamentos más prescritos estuvieron relacionados con el aparato digestivo y con terapia antiinfecciosa, principalmente $\beta$-lactámicos (penicilinas y cefalosporinas). Estos datos concuerdan con otros publicados previamente. ${ }^{15}$ En contraste, los fármacos más utilizados fuera de indicación fueron, primero, hipnóticos y sedantes; segundo, antitrombóticos; y tercero, antibióticos.

\section{Limitaciones del estudio}

En primer lugar, las que derivan de su diseño ya que se trata de un estudio retrospectivo y observacional. En segundo lugar, solo se analizaron ensayos clínicos llevados a cabo exclusivamente en niños; no se evaluaron los que incluían población adulta y pediátrica conjuntamente. Por último, las prescripciones manuales sobre la hoja de enfermería no se analizaron por falta de registro en el programa de PE.

\section{CONCLUSIÓN}

En nuestro centro, desde 2007, se produjo un incremento de los ECP, la mayoría de los cuales investigaron fármacos ya disponibles utilizados previamente fuera de FT. Los estudios no aleatorizados ni controlados fueron los más frecuentes. Además, la POMN se mantuvo constante.

\section{BIBLIOGRAFÍA}

1. Shah SS, Hall M, Goodman DM, Feuer P, et al. Off label drug use in hospitalized children. Arch Pediatr Adolesc Med 2007;161(3):282-90.

2. ShahS,Whittle A,Wildford B,GenslerG,Wenler D. How do institutional review boards apply the federal risk and benefit standards for pediatric research? JAMA 2004;291(4):476-82.

3. Neubert A, Wong IC, Bonifazi A, CatapanoM, etal. Defining off-label and unlicensed use of medicines for children: Results of a delphi survey. Pharmacol Res 2008;58(5-6):316-22.

4. Giglio ND, Malozowski S. Prescripciones fuera de prospecto. Arch Argent Pediatr 2004;102(2):121-4.

5. Li JS, Cohen-Wolkowiez M, Pasquali S. Pediatric cardiovascular drug trials, lessons learned. J Cardiovasc Pharmacol 2011;58(1):4-8.

6. Fernández-Llamazares CM, Manrique-Rodríguez S, Sanjurjo-Sáez M. Seguridad en el uso de medicamentos en pediatría. Arch Argent Pediatr 2011;109(6):510-8.

7. World Health Assembly (ed.). Resolución WHA60.20: Better medicines for children. Ginebra, Suiza: World Health Organization; 2007. [Consulta: 4 de febrero de 2014]. [Disponible en: http://www.who.int/childmedicines/ publications/WHA6020.pdf].

8. The Pediatric Research Equity Act of 2013, S.650. 108th Congress of the United States of America, first session, Washington, January 2003.

9. Reglamento del Parlamento Europeo y del Consejo sobre Medicamentos para Uso Pediátrico, 1901/2006 (12 de diciembre, 2006). [Consulta: 4 de febrero de 2014]. [Disponible en: http://www.ub.edu/legmh/disposici/ reg1901.htm].

10. Sampson MR, Benjamin DK, Cohen-Wolkowiez M. Evidence-based guidelines for pediatric clinical trials: focus on StaR Child Health. Expert Rev Clin Pharmacol 2012;5(5):525-31.

11. Hsein L, Breddemann A, Frobel AK, Heusch A, et al. Offlabel drug use among hospitalized children: identifying areas with the highest need for research. Pharm World Sci 2008;30(5):497-502.

12. Knopf H, Wolf IK, Sarganas G, Zhuang W, et al. Offlabel medicine use in children and adolescents: results of population-based study in Germany. BMC Public Health 2013;13(1):631.

13. Pandolfini C, Bonati M. European paediatric research and children's therapeutic needs. A trial review. Acta Paediatr 2008;97(9):1232-7.

14. Revised provisional priority list for studies into off-patent paediatric medicinal products. [Consulta: 8 de agosto de 2013]. [Disponible en: http://www.ema.europa. eu/docs/en_GB/document_library/Other/2013/06/ WC500143970.pdf].

15. Santos DB, Clavenna A, Bonati M, Coelho HL. Off-label and unlicensed drug utilization in hospitalized children in Fortaleza, Brazil. Eur J Clin Pharmacol 2008;64(11):1111-8. 